\title{
Probabilistic Forecasts of Solar Irradiance by Stochastic Differential Equations
}

\author{
Iversen, Jan Emil Banning; Morales González, Juan Miguel; Møller, Jan Kloppenborg; Madsen, Henrik
}

\section{Published in:}

Environmetrics

Link to article, DOI:

10.1002/env.2267

Publication date:

2014

Document Version

Early version, also known as pre-print

Link back to DTU Orbit

Citation (APA):

Iversen, J. E. B., Morales González, J. M., Møller, J. K., \& Madsen, H. (2014). Probabilistic Forecasts of Solar Irradiance by Stochastic Differential Equations. Environmetrics, 25(3), 152-164. https://doi.org/10.1002/env.2267

\section{General rights}

Copyright and moral rights for the publications made accessible in the public portal are retained by the authors and/or other copyright owners and it is a condition of accessing publications that users recognise and abide by the legal requirements associated with these rights.

- Users may download and print one copy of any publication from the public portal for the purpose of private study or research.

- You may not further distribute the material or use it for any profit-making activity or commercial gain

- You may freely distribute the URL identifying the publication in the public portal 
Environmetrics 00, 1-22

DOI: 10.1002/env.XXXX

\title{
Probabilistic Forecasts of Solar Irradiance by Stochastic Differential Equations
}

\author{
E. B. Iversen ${ }^{\mathrm{a} *}$ J. M. Morales ${ }^{\mathrm{a}}$, J. K. Møller ${ }^{\mathrm{a}}$, H. Madsen ${ }^{\mathrm{a}}$
}

Summary: Probabilistic forecasts of renewable energy production provide users with valuable information about the uncertainty associated with the expected generation. Current state-of-the-art forecasts for solar irradiance have focused on producing reliable point forecasts. The additional information included in probabilistic forecasts may be paramount for decision makers to efficiently make use of this uncertain and variable generation. In this paper, a stochastic differential equation (SDE) framework for modeling the uncertainty associated with the solar irradiance point forecast is proposed. This modeling approach allows for characterizing both the interdependence structure of prediction errors of short-term solar irradiance and their predictive distribution. A series of different SDE models are fitted to a training set and subsequently evaluated on a one-year test set. The final model proposed is defined on a bounded and time-varying state space with zero probability almost surely of events outside this space.

Keywords: Forecasting; Stochastic differential equations; Solar power; Probabilistic forecast;

Predictive distributions.

\section{INTRODUCTION}

The operation of electric energy systems is today challenged by the increasing level of uncertainty in the electricity supply brought in by the larger and larger share of renewables in the generation mix. Decision-making, operational and planning problems in electricity markets can be characterized by time-varying and asymmetric costs. These asymmetric costs are caused by the need to continuously balance the electricity system to guarantee

\footnotetext{
a Technical University of Denmark, Asmussens Alle, building 322, DK-2800 Lyngby, Denmark.

*Correspondence to: Mr. E. B. Iversen, Technical University of Denmark, Asmussens Alle, building 322, DK-2800 Lyngby, Denmark. Phone: +45606719 85, E-mail: jebi@dtu.dk
}

This paper has been submitted for consideration for publication in Environmetrics 
a reliable and secure supply of power. An understanding of the underlying uncertainty is, therefore, essential to satisfactorily manage the electricity system. This introduces the need for forecasts describing the entire variation of the renewable generation.

Solar irradiance is a source of renewable energy and, along with wind and hydro, is taking shape as a potential driver for a future free of fossil fuels. The worldwide installed capacity of photovoltaic energy systems has seen a rapid increase from 9.5 GW in 2007 to more than 100 GW by the end of 2012 (European Photovoltaic Industry Association (2013)). The energy generation from solar irradiance is subject to weather conditions and, as such, it constitutes a variable and uncertain energy source.

Current state-of-the-art forecasts for solar energy have focused on point forecasts, that is, the most likely or the average outcome. Such point forecasts, however, do not adequately describe the uncertainty of the power production. This is recognized by the abundance of significant works on probabilistic forecasting for wind power, see for ex. Pinson et al. (2007) and Zhou et al. (2013).

In the literature, a variety of different approaches have been taken to provide reliable solar power forecasts, based on time series (Huang et al. (2013), Boland (2008), Ji and Chee (2011), Bacher et al. (2009), Yang et al. (2012)), Artificial Neural Networks (Mihalakakou et al. (2000), Chen et al. (2011)), cloud motion forecasts (Perez et al. (2010)) and statistical models (Ridley et al. (2010), Kaplanis and Kaplani (2010)). A review of some of these approaches is found in Pedro and Coimbra (2012). In Chen et al. (2011) Artifical Neural Networks are used in combination with a weather type classification, to provide point forecasts of PV production. In Lorenz et al. (2009), a forecast method that makes use of a clear sky model and numerical weather predictions is developed, also accounting for orientation and tilt of the PV panel. The paper by Bhardwaj et al. (2013) introduces a hidden Markov model for solar irradiance based on fuzzy logic. They exploit inputs such as humidity, temperature, 
air pressure and wind speed, among others. A time-series model for predicting one-hourahead solar power production is considered in Yang et al. (2012). This paper employs a cloud cover index to model the absorption and refraction of the incoming light through the atmosphere. In Bacher et al. (2009), an auto-regressive model with exogenous input is proposed. It predicts weighing the past observations and the numerical weather prediction and introduces a clear sky model to capture the diurnal variation.

Probabilistic forecasting of solar irradiance is, though, in its infancy. One work in this area is the one by Mathiesen et al. (2013), where post-processing of numerical weather predictions are applied to obtain probabilistic forecasts. Previous work on stochastic differential equations and solar irradiance is, to the best of our knowledge, limited to Soubdhan and Emilion (2010), which formulates a very simple stochastic differential equation model for solar irradiance. As a consequence of its simplicity, the model was largely unsuccessful at forecasting. Stochastic differential equations are fruitfully used for wind power forecasting in Møller et al. (2013) by considering state-dependent diffusions and external input.

This paper describes a new approach to solar irradiance forecasting based on stochastic differential equations (SDEs). Modeling with SDEs has multiple benefits, among others:

- SDE models are able to produce reliable point forecasts as well as probabilistic forecasts.

- Model extensions are easy to formulate and have an intuitive interpretation. We can start with a simplistic model and extend it to a sufficient degree of complexity.

- We can model processes that are bounded and assign zero probability to events outside the bounded interval, which is essential for correct probabilistic forecasts of solar irradiance.

- We leave the discrete-time realm of Gaussian innovations and consider instead the more general class of continuous-time processes with continuous trajectories.

- SDEs span a large class of stochastic processes with classical time-series models as special cases. 
The rest of this paper is structured as follows: Section 2 gives a general introduction to the stochastic differential equation framework and describes an estimation procedure. Section 3 starts with a simple SDE model. secondly we propose a mechanistic model which captures some of the key physical qualities of solar irradiance. Lastly, in Section 3, we provide a SDE model that extends the mechanistic model to overcome some statistical deficiencies. In Section 4, the different models are compared to simple as well as complex benchmarks and the performance of the finished model is assessed. Lastly, Section 5 concludes the paper.

\section{STOCHASTIC DIFFERENTIAL EQUATIONS}

Suppose that we have the continuous time process $X_{t} \in \mathcal{X} \subset \mathbb{R}^{n}$. In general, it is only possible to observe continuous time processes in discrete time. We observe the process $X_{t}$ through an observation equation at discrete times. Denote the observation at time $t_{k}$ by $Y_{k} \in \mathcal{Y} \subset \mathbb{R}^{l}$ for $k \in\{0, \ldots, N\}$. Let the observation equation be given by:

$Y_{k}=h\left(X_{t_{k}}, t_{k}, e_{k}\right)$

where the variable $t_{k}$ allows for dependence on an external input at time $t_{k}, e_{k} \in \mathbb{R}^{l}$ is the random observation error, and $h(\cdot) \in \mathbb{R}^{l}$ is the function that links the process state to the observation. The simplest form of the observation equation is $h(\cdot)=X_{t_{k}}+e_{k}$.

\subsection{Definition of Stochastic Differential Equations}

In the ordinary differential equation setting, the evolution in time of the state variable $X_{t}$ is given by the deterministic system equation

$\frac{d X_{t}}{d t}=f\left(X_{t}, t\right)$ 
where $t \in \mathbb{R}$ and $f(\cdot) \in \mathbb{R}^{n}$. Complex systems such as weather systems are subject to random perturbations of the input or processes that are not specified in the model description. This suggests introducing a stochastic component in the state evolution to capture such perturbations. This can be done by formulating the state evolution as a stochastic differential equation (SDE), as done in Øksendal (2010). Thus, we can formulate the time evolution of the state of the process by the form:

$\frac{d X_{t}}{d t}=f\left(X_{t}, t\right)+g\left(X_{t}, t\right) W_{t}$

where $W_{t} \in \mathbb{R}^{m}$ is an $m$-dimensional standard Wiener process and $g(\cdot) \in \mathbb{R}^{n \times m}$ is a matrix function (Øksendal, 2010). Multiplying with $d t$ on both sides of (3) we get the standard SDE formulation:

$d X_{t}=f\left(X_{t}, t\right) d t+g\left(X_{t}, t\right) d W_{t}$

Notice that we allow for a complex dependence on $t$, including external input at time $t$. While this form is the most common for SDEs, it is not well defined, as the derivative of $W_{t}, d W_{t}$, does not exist. Instead, it should be interpreted as an informal way of writing the integral equation:

$$
X_{t}=X_{0}+\int_{0}^{t} f\left(X_{s}, s\right) d s+\int_{0}^{t} g\left(X_{s}, s,\right) d W_{s}
$$

In Equation (5), the behavior of the continuous time stochastic process $X_{t}$ is expressed as the sum of an initial stochastic variable, an ordinary Lebesgue integral, and an Ito integral. In a deterministic ordinary differential equation setting, the solution would be a single point for each future time $t$. In the SDE setting, in contrast, the solution is the probability density of $X_{t}$ for any state, $x$, and any future time, $t$. For an Itō process given by the stochastic differential equation defined in (4) with drift $f\left(X_{t}, t\right)$ and diffusion coefficient $g\left(X_{t}, t\right)=\sqrt{2 D\left(X_{t}, t\right)}$, the probability density $j(x, t)$ in the state $x$ at time $t$ of the random 
variable $X_{t}$ is given as the solution to the partial differential equation known as the FokkerPlanck equation (Björk, 2009):

$\frac{\partial}{\partial t} j(x, t)=-\frac{\partial}{\partial x}[f(x, t) j(x, t)]+\frac{\partial^{2}}{\partial x^{2}}[D(x, t) j(x, t)]$.

Thus, given a specific SDE, we can find the density at any future time by solving a partial differential equation.

SDEs are a general class of processes. This is stated by the Lévy-Itō decomposition, which says that, under sufficient regularity conditions, all stochastic processes with continuous trajectories can be written as SDEs (Øksendal, 2010). Hence many of the ordinary discretetime stochastic processes can be seen as a SDE being sampled at discrete times, and therefore, SDEs is a generalization of generic time-series models in discrete time. Other useful introductions to SDEs are among others Kloeden and Pearson (1977) and Mikosch (1998).

\subsection{Parameter Estimation}

In this section, we outline how to estimate parameters in a SDE of a general form and, in particular, with a state-dependent diffusion term. A thorough description of the procedure used here (and other estimation procedures) is found in Jazwinski (2007). First, we go into detail on the estimation procedure of the parameters of a SDE with a state-independent diffusion term. Second, we show how to transform a process with state-dependent diffusion term into a process with a unit diffusion term, whereby the previously mentioned estimation procedure can be applied.

Consider the model defined by Equations (1) and (4) given by:

$$
\begin{aligned}
d X_{t} & =f\left(X_{t}, t\right) d t+g\left(X_{t}, t\right) d W_{t} \\
Y_{k} & =h\left(X_{t_{k}}, t_{k}, e_{k}\right) .
\end{aligned}
$$


On the basis that we want to estimate the parameters in the above model, the problem can be formulated as follows: Find a parameter vector, $\hat{\theta} \in \Theta$, that maximizes some objective function of $\theta$. There are several possible choices for an objective function. A natural choice in this framework is to choose an objective function that maximizes the probability of seeing the observations given by $\mathcal{Y}_{N}=\left\{Y_{0}, \ldots, Y_{N}\right\}$. This leads to choosing the likelihood function as objective function, i.e.,

$L\left(\theta ; \mathcal{Y}_{N}\right)=p\left(\mathcal{Y}_{N} \mid \theta\right)=\left(\prod_{k=1}^{N} p\left(Y_{k} \mid \mathcal{Y}_{k-1}, \theta\right)\right) p\left(Y_{0} \mid \theta\right)$

Even though this problem could, in principle, be solved using the Fokker-Planck equation, this is only feasible for systems with simple structures, as it involves solving a complex partial differential equation.

The estimation procedure, which we shall introduce next, relies on the system having a specific form, namely:

$$
\begin{aligned}
d X_{t} & =f\left(X_{t}, t\right) d t+g(t) d W_{t} \\
Y_{k} & =h\left(X_{t_{k}}, t_{k}\right)+e_{k} .
\end{aligned}
$$

In the system defined by Equations (10) and (11), we assume that $g(\cdot) \in \mathbb{R}^{n \times n}$ does not depend on the state $X_{t}$. Also, we assume that the observation noise is an additive Gaussian white noise, i.e., $e_{k} \sim \mathcal{N}\left(0, S_{k}\left(t_{k}\right)\right)$, where $S_{k}\left(t_{k}\right)$ is some covariance matrix, possibly depending on time. It is clear that restricting $g(\cdot)$ to not depend on $X_{t}$ limits our model framework severely. As we shall see, this can, to some degree, be remedied by a transformation using Itō-calculus. The restriction of having additive Gaussian measurement noise should be dealt with by transformations of the observations.

As the system defined by Equations (10) and (11) is driven by Wiener noise, which has Gaussian increments, and the observation noise is Gaussian, it is reasonable to assume that the density of $Y_{k} \mid \mathcal{Y}_{k-1}$ can be approximated by a Gaussian distribution. Note that the 
Gaussian distribution is completely characterized by its mean and covariance. This implies that using the extended Kalman filter, which is linear, is appropriate.

The one-step predictions for the mean and variance are defined as:

$$
\begin{aligned}
\widehat{Y}_{k \mid k-1} & =\mathbb{E}\left[Y_{k} \mid \mathcal{Y}_{k-1}, \theta\right] \\
R_{k \mid k-1} & =\mathbb{V}\left[Y_{k} \mid \mathcal{Y}_{k-1}, \theta\right]
\end{aligned}
$$

where $\mathbb{E}[\cdot]$ and $\mathbb{V}[\cdot]$ denote the expectation and variance, respectively. The innovation is given by

$\epsilon_{k}=Y_{k}-\widehat{Y}_{k \mid k-1}$

Using this, we can now write the likelihood function as

$L\left(\theta ; \mathcal{Y}_{N}\right)=\left(\prod_{k=1}^{N} \frac{\exp \left(-\frac{1}{2} \epsilon_{k}^{\top} R_{k \mid k-1}^{-1} \epsilon_{k}\right)}{\sqrt{\operatorname{det}\left(R_{k \mid k-1}\right)}(\sqrt{2 \pi})^{l}}\right) p\left(Y_{0} \mid \theta\right)$

where $l$ is the dimension of the sample space and $(\cdot)^{\top}$ denotes the vector transpose. The estimate of $\theta$ can be found by solving the optimization problem

$\hat{\theta}=\arg \max _{\theta \in \Theta}\left(\log \left(L\left(\theta ; \mathcal{Y}_{N}\right)\right)\right)$

The Kalman gain governs how much the one-step prediction of the underlying state, $\widehat{X}_{k \mid k-1}$, should be adjusted to form the state update, $\widehat{X}_{k \mid k}$, from the new observation. This is given by

$K_{k}=P_{k \mid k-1} C^{\top} R_{k \mid k-1}^{-1}$ 
where $C$ is the first order expansion of $h(\cdot)$, i.e., the Jacobian, and $P_{k \mid k-1}$ is the covariance of the one-step prediction. The state update is then given by

$$
\begin{aligned}
\widehat{X}_{k \mid k} & =\widehat{X}_{k \mid k-1}+K_{k} \epsilon_{k} \\
P_{k \mid k} & =P_{k \mid k-1}-K_{k} R_{k \mid k-1}^{-1} K_{k}^{\top}
\end{aligned}
$$

Hence, the state update is a combination of the previous state estimate and the new information obtained from the $k$ 'th observation, $Y_{k}$.

The procedure of estimating the parameters is currently being implemented as an $\mathrm{R}$ package and is described in Juhl et al. (2013). The estimation procedure is done in the statistical software R. we use the extended Kalman filter as provided by Juhl et al. (2013) to give predictions of future observation and implement the likelihood function directly in $\mathrm{R}$. The likelihood function is then optimized using the nlminb routine for constrained optimization.

\subsection{Itō Calculus and the Lamperti Transform}

We will now discuss how a SDE of the form in Equation (7) can be transformed to the form in Equation (10) to allow for the estimation procedure previously introduced. The fundamental tool for the transformation of SDEs is Itō's lemma, as stated in Øksendal (2010). Below we introduce the 1-dimensional Itō formula and the Lamperti transform. The multidimensional Itō formula is covered in Øksendal (2010). For a more detailed description of the Lamperti transform and how to apply it to multivariate processes, see Møller and Madsen (2010).

Theorem 1 (The 1-dimensional Itō formula ) Let $X_{t}$ be an Itō process given by

$$
\begin{aligned}
& d X_{t}=f\left(X_{t}, t\right) d t+g\left(X_{t}, t\right) d W_{t} . \\
& \text { Let } \psi(x, t) \in C^{2}([0, \infty)) \times \mathbb{R} . \text { Then } \\
& Z_{t}=\psi\left(X_{t}, t\right)
\end{aligned}
$$


is again an Itō process, and

$d Z_{t}=\frac{\partial \psi}{\partial t}\left(X_{t}, t\right) d t+\frac{\partial \psi}{\partial x}\left(X_{t}, t\right) d X_{t}+\frac{1}{2} \frac{\partial^{2} \psi}{\partial x^{2}}\left(X_{t}, t\right)\left(d X_{t}\right)^{2}$

where $\left(d X_{t}\right)^{2}$ is calculated according to the rules

$d t \cdot d t=d t \cdot d W_{t}=d W_{t} \cdot d t=0, \quad d W_{t} \cdot d W_{t}=d t$

The Itō formula stated in Theorem 1 can be used to transform the process to a SDE with unit diffusion by the Lamperti transform.

Theorem 2 (Lamperti transform) Let $X_{t}$ be an Itō process defined as in (20), and define

$\psi\left(X_{t}, t\right)=\left.\int \frac{1}{g(x, t)} d x\right|_{x=X_{t}}$

If $\psi$ represents a one to one mapping from the state space of $X_{t}$ onto $\mathbb{R}$ for every $t \in[0, \infty)$, then choose $Z_{t}=\psi\left(X_{t}, t\right)$. Then $Z_{t}$ is governed by the $S D E$

$$
\begin{aligned}
d Z_{t}= & \left(\psi_{t}\left(\psi^{-1}\left(Z_{t}, t\right), t\right)+\frac{f\left(\psi^{-1}\left(Z_{t}, t\right), t\right)}{g\left(\psi^{-1}\left(Z_{t}, t\right), t\right)}\right. \\
& \left.-\frac{1}{2} g_{x}\left(\psi^{-1}\left(Z_{t}, t\right), t\right)\right) d t+d W_{t}
\end{aligned}
$$

where $g_{x}(\cdot)$ and $g_{t}(\cdot)$ denote the derivatives of $g(\cdot)$ with regard to $x$ and $t$, respectively, and $\psi_{t}$ denotes the derivative of $\psi$ with respect to $t$.

This result is obtained by applying the Itō formula.

\section{SOLAR IRRADIANCE}

In this section we apply the theory in Section 2 to build a model for solar irradiance. The approach is to start out with a simple model and extend it until we reach a model that captures the dynamics adequately. Firstly we extend the simple model from a mechanistic 
understanding of solar irradiance, for instance that solar irradiance is a bound process and our dynamical model should reflect this. Secondly, we extend the mechanistic model by identifying statistical deficiencies and extending the model to resolve those. Thus, we should end up with a model, that captures the mechanics of the system and is statistically sound.

\subsection{Data}

The data set at our disposal belongs to a meteorological station located in the western part of Denmark. The data include hourly observations of irradiance on a flat surface together with predictions for irradiance based on a numerical weather prediction model from the Danish Meteorological Institute. The numerical weather prediction (NWP) provides a 48hour forecast of the irradiance, which is updated every 6 hours. We use the most recent forecast in the model. The data covers a period of three years from 01/01-2009 to 31/122011. We divide the period into a training and a test set, with the training set covering the first two years and the test set the last year.

\subsection{Model 1: A Simple Model Tracking the NWP}

We start by introducing a simple SDE model for solar irradiance that tracks the numerical weather prediction provided by the Danish Meteorological Institute, i.e.,

$$
\begin{aligned}
d X_{t} & =\theta_{x}\left(p_{t} \mu_{x}-X_{t}\right) d t+\sigma_{x} d W_{t} \\
Y_{k} & =X_{t_{k}}+\epsilon_{k} .
\end{aligned}
$$

In this model and the following, we denote the observed solar irradiance at time $t_{k}$ by $Y_{k}$. $p_{t}$, is an external input representing the predicted irradiance at time $t$. In the model, we have parameter $\mu_{x}$, which allows for a local scaling of the $p_{t}$, such that it does not over or under shoot on average. The parameter $\theta_{x}$ determines how rapidly the model reverts to the 
predicted level of irradiance. The system noise is controlled by parameter $\sigma_{x}$. The observation error is denoted $\epsilon_{k}$ and is a stochastic variable with distribution $\mathcal{N}\left(0, \sigma_{\epsilon}\right)$.

\subsection{Model 2: A Mechanistic Extension}

Some physical characteristics of solar irradiance are clearly not captured in Model 1. Among these are issues related to the bounded nature of solar irradiance: It is never below zero and also never above some upper bound determined by the light emitted by the sun and the season. We will remedy this issue and others with the following model:

$$
\begin{aligned}
d X_{t} & =\theta_{x}\left(\frac{p_{t}+\beta_{x}}{\gamma m_{t}+\delta} \mu_{x}-X_{t}\right) d t+\sigma_{x} X_{t}\left(1-X_{t}\right) d W_{t} \\
Y_{k} & =\gamma m_{t_{k}} X_{t_{k}}+\epsilon_{k} .
\end{aligned}
$$

Solar irradiance is highly cyclical. To capture this, we extend the simple SDE model defined in (27)-(28) by introducing the maximum irradiance in hour $t, m_{t}$, as a scaling factor. Here we compute $m_{t}$ according to Bird and Hulstrom (1981), where the refraction and absorption in the atmosphere is set to zero. This leads to a formulation where we let the stochastic process $X_{t}$ denote the proportion of extra terrestrial irradiance (i.e., the irradiance that would arrive at the surface if there were no atmosphere) that reaches the surface.

The above process is, however, undefined at night, when $m_{t}=0$. To overcome this, we can instead think of $X_{t}$ as a process that describes the state of the atmosphere and how much solar irradiance there would potentially be allowed through. In this context, it clearly makes sense to have $X_{t}$ defined at night. Thus, we can solve the issue of having $m_{t}=0$ by adding a small constant, say $\delta=0.01$. Given that the $p_{t}$ is also equal to zero at night, we introduce another parameter $\beta_{x}$ (to be estimated) that is added to $p_{t}$ such that $X_{t}$ is not forced to tend to zero at night.

Also we note that in this set-up the proportion of solar irradiance that reaches the surface is naturally bounded to be between zero and one. Thus we want to introduce bounds for 
the process $X_{t}$. This is easily done by introducing a state-dependent diffusion, where the diffusion term decreases to zero as the process approaches the bounds. This implies that the drift term takes over near the bounds. Furthermore, since we assume that $\theta_{x}>0$ and have that $1>\frac{p_{t}+\beta_{x}}{m_{t}+\delta}>0$, the drift term eventually pulls the process away from the bounds.

We have assumed the extra terrestrial irradiance, $m_{t}$, as the upper limit for the solar irradiance. It should be clear, however, that this level can never be attained, because there will always be some refraction by the atmosphere. Hence, we can possibly scale down the upper limit, $m_{t}$, to improve the model. This is done by introducing a factor, $\gamma$, on the maximum solar irradiance. An improvement of the model would be to consider the air mass Kasten and Young (1989), which takes care of the fact that the distance the light travels through the atmosphere is longer, when the sun is near the horizon. This, however, will be left to future work.

In the estimation procedure we have assumed that the noise is non-state dependent, which is clearly not the case here. Therefore, we need to work with the Lamperti transformed process. The Lamperti transformation is given by:

$$
\begin{aligned}
& Z_{t}=\psi\left(X_{t}, t\right)=\left.\int \frac{1}{\sigma_{x} x(1-x)} d x\right|_{x=X_{t}}=-\frac{1}{\sigma_{x}} \log \left(\frac{1-x}{x}\right) \\
& X_{t}=\psi^{-1}\left(Z_{t}, t\right)=\frac{1}{1+e^{-\sigma_{x} Z_{t}}} .
\end{aligned}
$$

Noting that $\psi_{t}(\cdot)=0$ and $g_{x}(x, t)=\sigma_{x}(1-2 x)$, we can now make use of the Lamperti transform to obtain the process on the transformed $Z$-space, which becomes:

$$
\begin{aligned}
d Z_{t} & =\left(\frac{\theta_{x}\left(\frac{p_{t}+\beta_{x}}{\gamma m_{t}+\delta} \mu_{x}-\frac{1}{1+e^{-\sigma_{x} Z_{t}}}\right)}{\sigma_{x} \frac{1}{1+e^{-\sigma_{x} Z_{t}}}\left(1-\frac{1}{1+e^{-\sigma_{x} Z_{t}}}\right)}-\frac{\sigma_{x}}{2}\left(1-2 \frac{1}{1+e^{-\sigma_{x} Z_{t}}}\right)\right) d t+d W_{t} \\
Y_{k} & =\gamma m_{t_{k}} \frac{1}{1+e^{-\sigma_{x} Z_{t}}}+\epsilon_{k} .
\end{aligned}
$$

In the sequel, we shall only state the model in the original domain and not in the Lamperti transformed domain, as they are equivalent in the sense of yielding the same output 
[Figure 1 about here.]

In this mechanistic model, we want to consider any deficiencies to improve. This can, among other approaches, be done by considering the autocorrelation of the studentized residuals. In Figure 1 we show the autocorrelation of the studentized residuals of Model 2. We see that there are significant lags at the first lags and that there is some periodicity in the lags. This points in the direction of some statistical improvements that can be made to the model, which we consider next in Model 3.

\subsection{Model 3: Statistical Extensions}

How fast $X_{t}$ is attracted to its predicted level may vary over time. This may be due to the NWP being more accurate at some times than at others. Furthermore, there is a lag in the numerical weather prediction as it takes 4 hours to solve the NWP model. As the NWP model is only run every 6 hours, this leads to the NWP being between 4 and 10 hours old, which may also cause us to have varying confidence in the NWP.

To address this issue, we have introduced the stochastic process $A_{t}$, which reverts to the level $\mu_{A}$. The speed at which this reversion occurs is determined by $\theta_{A}$, while the system noise is governed by $\sigma_{A}$. $A_{t}$ governs how rapidly $X_{t}$ tends to its predicted level. Also, there might be variations over the day in the accuracy of the numerical weather prediction. This leads to the following model:

$$
\begin{aligned}
d X_{t}= & e^{A_{t}}\left(\frac{p_{t}+\beta_{x}}{\gamma m_{t}+\delta}\left(\mu_{x}-\omega_{1} \sin \left(\frac{2 \pi}{24} t+\omega_{2}\right)\right)-X_{t}\right) d t \\
& +\sigma_{x} X_{t}\left(1-X_{t}\right) d W_{1, t} \\
d A_{t}= & \theta_{A}\left(\mu_{A}-A_{t}\right) d t+\sigma_{A} d W_{2, t} \\
Y_{k}= & \gamma m_{t_{k}} X_{t_{k}}+\epsilon_{k} .
\end{aligned}
$$

Firstly, note that we use a sinusoid to describe a periodic behaviour. Secondly, we work in 
hourly time steps, which explains $\frac{2 \pi}{24} t$. We then introduce a period shift, $\omega_{2}$, and an amplitude, $\omega_{1}$. The sinusoid is added to the scaling of the meteorological prediction, which translates into the $p_{t}$ being more accurate in some hours of the day than in others.

We have ended up with a model that includes a maximum hourly irradiance, a numerical weather prediction as external input, stochastic time constants, and a non-Gaussian system noise that confines the process between zero and the extra terrestrial irradiance. In the following section, validation results of the final model are presented.

\section{MODEL VALIDATION}

In this section, the different models are fitted to the data pertaining to the training set and evaluated in terms of their likelihood and information criteria.

In terms of computational costs, we have done the computations on a network mainframe with $201.8 \mathrm{GHz}$ processors with a partial paralellization of the optimization problem. In this setup and the models fitted to the test set of two years of hourly data Model 1 is optimized in less than 3 minutes, Model 2 is optimized in just under 16 minutes and the fitting of Model 3 is completed 41 minutes. It should be noted, however, that the paralellization has diminishing returns to scale. For comparison we have fitted Model 3 on a personal computer with a $2.7 \mathrm{GHz}$ processor and $8 \mathrm{~GB}$ of ram in just over 2 hours.

[Table 1 about here.]

As we propose a model for solar irradiance based partially on physical characteristics of the system, a first step in validating the model is to consider if the parameter estimates are feasible in terms of the physics of the system. Specifically, from the physics of the system, we would expect $\mu_{x}$ around $1, \beta_{x}$ to be smaller than 0.01 and $\gamma$ close to, but smaller than 1. We see that all these conditions are satisfied. 
To compare the models with more classical alternatives, we consider an autoregressive model with external input (ARX) and an autoregressive model with external input and time-varying system variability, where the prediction variance is modelled using a generalized linear model (ARX-GLM). The AR model is specified as follows:

$Y_{k}=\psi_{0}+\sum_{i=1}^{p} \psi_{i} Y_{k-i}+\epsilon_{k}, \quad$ where $\epsilon_{k} \sim \mathcal{N}\left(0, \sigma^{2}\right)$.

The ARX model takes the form

$Y_{k}=\psi_{0}+\sum_{i=1}^{p} \psi_{i} Y_{k-i}+\phi p_{k}+\epsilon_{k}, \quad \epsilon_{k} \sim \mathcal{N}\left(0, \sigma^{2}\right)$

The ARX-GLM model is specified as

$Y_{k}=\psi_{0}+\sum_{i=1}^{p} \psi_{i} Y_{k-i}+\phi p_{k}+\epsilon_{k}, \quad \tilde{\epsilon}_{k+1} \sim \mathcal{N}\left(0, f_{\tilde{\epsilon}}(\cdot)^{2}\right)$

We find, after a fitting procedure, that an appropriate form of the variance scaling in the generalized linear model is

$f_{\tilde{\epsilon}}(k+1)=\sigma\left(m_{t_{k+1}}\right)^{3 / 4}$,

where $m_{t_{k+1}}$ is specified as in Model 2. For a general introduction to generalized linear models see Madsen and Thyregod (2011).

Additionally, we benchmark against climatological forecasts. The naïve forecast method is to use the empirical distribution, with no time dependence, to predict the solar irradiance. A slightly less naïve approach is to use the empirical distribution of irradiance as a function of hour-of-day. A third climatological benchmark is to use both hour-of-day and month-ofyear to predict the distribution. These benchmarks are clearly naïve as they do not use the previous observation to predict. Also, as the climatological approach is non-parametric, we use the empirical likelihood, see for instance Bera and Bilias (2002), to evaluate the fit. 
[Table 2 about here.]

The results of the different models are presented in Table 2. When computing the likelihood values, we let only observations during the day contribute with likelihood. This is done as to not obstruct the picture by fitting solar irradiance models at night. This holds true both for the benchmarks as well as the fitted SDE models. We see that Model 3 best describes the data in the training set, as well as in the test set. Furthermore note that the improvement from the quite naïve Model 1 to Model 2 is huge, which justifies the change in the state space.

[Figure 2 about here.]

An important analysis tool is the autocorrelation function. We compute this function for the studentized residuals. The autocorrelation for different lags for the different models is shown in Figure 2. Observe that the range of the y-axis is $(-0.05,0.25)$ to better show the significance levels for the different lags. In Figure 2, we see that Model 1 has many autocorrelation coefficients that are significant, indicating that this model clearly does not properly capture the dynamics of the solar irradiance process. In contrast, for Model 2, only a couple of the first lags along with lags around lag 24 are still significant. We then end up with model 3, in which the first 22 lags are insignificant in predicting the next time-step. Notice, however, that around lag number 24, we again begin to see significant autocorrelation coefficients. This is most likely caused by local conditions like shadowing (by trees or buildings) or local recurrent weather phenomena such as sea breeze (Bacher et al. $(2013))$.

[Figure 3 about here.]

The output of the SDE models is the conditional predictive density at each point in time. We obtain these predictive densities by Monte Carlo simulation, however, other numerical procedures are also possible such as solving equation (6). In Figure 3 the observations are 
shown along with the predictive densities given by Model 3, with warmer colors having higher probability. Initially we may note that the conditional density seems to cover the obsercations. Another feature of this model is that it assigns zero probability to events outside the state space, that is, for values of irradiance higher than the maximum or lower than zero. Notice, in the bottom plot of Figure 3, that the density spreads out, as here we represent predictions issued 24 hours ahead instead of 1 hour ahead. A further illustration of this is seen in Figure 4,

[Figure 4 about here.]

where the $95 \%$ prediction interval is shaded in gray. Note from this figure that this interval decreases as we approach the limits of the state space, that is, when the process comes closer to the maximum irradiance or to zero. Also, notice that the prediction interval is not symmetric around the point prediction, especially when approaching the limits. Upon careful inspection, it can be found that the prediction interval is the widest when we predict around $50 \%$, which is to be expected from the physics of the system. Besides, notice that the 24-hour ahead forecast has a wider $95 \%$ prediction interval.

To validate the accuracy of the predictive density, we can evaluate the predictive quantiles in the distribution. This is done by counting how many observations lie on each side of the predictive quantile in question and comparing it to the expected number.

[Table 3 about here.]

In Table 3 the exceedances of the predictive quantiles for Model 3 are shown. The predictive distribution is found by simulating the process via Monte Carlo simulation for a large number of trajectories. In a perfect data fit, the expected quantiles match the observed ones exactly. For the 1-hour prediction on the training set, we see an excellent performance, with the frequency of exceedences quite close to the expected one in a perfect fit.t For the 24-hour prediction horizon, we observe a slightly lower number of exceedances than expected. This is 
also true for the test set, especially for the 24-hour ahead quantiles. It should be taken into account hat Model 3 has been fitted on the basis of one-step-ahead predictions, that is, the prediction of the next hour on the training set. Thus, the model is not tuned to predictions for a 24-hour horizon, even though it seems to perform reasonably well.

[Figure 5 about here.]

To evaluate the long term behaviour of the fitted model we can compare the quantiles of the data with quantiles generated by simulating the model. This is done in Figure 5. We see that the empirical and theoretical quantiles lie on a somewhat straight line. We see that the performance on the training set is better than on the test set, as we would expect. The stationary distribution of the model on the test set, nevertheless, seems to be aligned with the empirical distribution.

\section{CONCLUDING REMARKS}

With the increasing penetration of renewable generation in energy systems, forecasting renewable production is becoming crucial for its efficient integration. Especially asymmetric costs in time requires an understanding of the uncertainty associated with the stochastic renewable production. An example of this is ex. the power market in the nordic countries, where power is sold 12-36 hours ahead of generation. If the seller is then not able to deliver, she is required to procure the power in balancing markets where the power is typically many times more expensive. This paper proposes a stochastic differential equation framework for modeling the uncertainty associated with solar irradiance. It allows us to model a process that is confined to a bounded state space by physical restrictions on the system. This can also be done by truncating distributions, however, internalizing it in the model dynamics provides a more realistic model with better performance and also it is useful for probabilistic forecasting. 
The starting point for the modeling done in this paper is a simple SDE that tracks the expected solar irradiance from a numerical weather prediction. By normalizing the weather prediction with the maximum irradiance, we can capture the periodic behaviour in the dynamics, and consequently, achieve major improvements. We can tune the diffusion term to model the actual behaviour of the process and confine it to a bounded interval. The SDE formulation allows for formulating complex model structures and to track conditional distributions at any point in time. Our proposed SDE modeling approach outperforms simple as well as more complex benchmarks.

Even though there is a relation between solar irradiance and produced power from a photovoltaic panel, such a relation is not trivial. It depends on tilt and orientation of the PV panel as well as on its efficiency, which may vary as the panel gets dirty or deteriorates over time. To address this, an adaptive estimation approach would be appropriate. Future studies will be directed at constructing models to capture this and produce probabilistic forecasts for solar power via a power curve. Since what is important to the energy system is the total input of renewable energy, future studies will also be directed at co-modeling wind and solar power, as these are expected to be main contributors to the energy mix of the future. Another potential line of future research is the modeling of the interdependence between the power output of solar farms at different locations.

\section{REFERENCES}

Bacher P, Madsen H, Nielsen H, 2009. Online short-term solar power forecasting. Solar Energy 83(10): $1772-1783$.

Bacher P, Madsen H, Perers B, Nielsen H, 2013. A non-parametric method for correction of global radiation observations. Solar Energy 88: 13 - 22.

Bera AK, Bilias Y, 2002. The mm, me, ml, el, ef and gmm approaches to estimation: a synthesis. Journal of Econometrics 107(1): 51-86. 
Bhardwaj S, Sharma V, Srivastava S, Sastry OS, Bandyopadhyay B, Chandel SS, Gupta JRP, 2013. Estimation of solar radiation using a combination of hidden Markov model and generalized fuzzy model. Solar Energy 93: 43-54.

Bird RE, Hulstrom RL, 1981. Simplified clear sky model for direct and diffuse insolation on horizontal surfaces. Technical report, Solar Energy Research Inst., Golden, CO (USA).

Björk T, 2009. Arbitrage Theory in Continuous Time. Oxford Finance Series, OUP Oxford.

Boland J, 2008. Time series modelling of solar radiation. In Badescu V (ed.), Modeling Solar Radiation at the Earth?s Surface, Springer Berlin Heidelberg, 283-312.

Chen C, Duan S, Cai T, Liu B, 2011. Online 24-h solar power forecasting based on weather type classification using artificial neural network. Solar Energy 85(11): 2856-2870.

European Photovoltaic Industry Association, 2013. Global market outlook for photovoltaics 2013-2017. Online.

Huang J, Korolkiewicz M, Agrawal M, Boland J, 2013. Forecasting solar radiation on an hourly time scale using a coupled autoregressive and dynamical system (cards) model. Solar Energy 87(1).

Jazwinski AH, 2007. Stochastic processes and filtering theory. Courier Dover Publications.

Ji W, Chee KC, 2011. Prediction of hourly solar radiation using a novel hybrid model of arma and tdnn. Solar Energy 85(5): 808-817.

Juhl R, Kristensen NR, Bacher P, Kloppenborg J, Madsen H, 2013. Ctsm-r user guide. Technical University of Denmark 2.

Kaplanis S, Kaplani E, 2010. Stochastic prediction of hourly global solar radiation for patra, greece. Applied Energy 87(12): 3748-3758.

Kasten F, Young AT, 1989. Revised optical air mass tables and approximation formula. Appl. Opt 28(22): 4735-4738.

Kloeden PE, Pearson R, 1977. The numerical solution of stochastic differential equations. The Journal of the Australian Mathematical Society. Series B. Applied Mathematics 20(01): 8-12.

Lorenz E, Hurka J, Heinemann D, Beyer HG, 2009. Irradiance forecasting for the power prediction of gridconnected photovoltaic systems. IEEE Journal of Selected Topics in Applied Earth Observations and Remote Sensing 2(1): 2-10.

Madsen H, Thyregod P, 2011. Introduction to General and Generalized Linear Models. Chapman \& Hall/CRC Texts in Statistical Science Series, CRC PressINC. 
Mathiesen P, Brown J, Kleissl J, 2013. Geostrophic wind dependent probabilistic irradiance forecasts for coastal california. IEEE Transactions on Sustainable Energy 4(2): 510-518.

Mihalakakou G, Santamouris M, Asimakopoulos D, 2000. The total solar radiation time series simulation in athens, using neural networks. Theoretical and Applied Climatology 66(3-4): 185-197.

Mikosch T, 1998. Elementary stochastic calculus: with finance in view, volume 6. World Scientific.

Møller J, Madsen H, 2010. From state dependent diffusion to constant diffusion in stochastic differential equations by the Lamperti transform. Technical report, Technical University of Denmark.

Møller J, Pinson P, Madsen H, 2013. Probabilistic forecasts of wind power generation by stochastic differential equation models. Technical report, Technical University of Denmark.

Øksendal B, 2010. Stochastic Differential Equations: An Introduction with Applications. Universitext (1979), Springer.

Pedro H, Coimbra C, 2012. Assessment of forecasting techniques for solar power production with no exogenous inputs. Solar Energy 86(7): 2017-2028.

Perez R, Kivalov S, Schlemmer J, Hemker Jr K, Renné D, Hoff TE, 2010. Validation of short and medium term operational solar radiation forecasts in the us. Solar Energy 84(12): 2161-2172.

Pinson P, Nielsen H, Møller J, Madsen H, Kariniotakis G, 2007. Non-parametric probabilistic forecasts of wind power: Required properties and evaluation. Wind Energy 10(6): 497-516.

Ridley B, Boland J, Lauret P, 2010. Modelling of diffuse solar fraction with multiple predictors. Renewable Energy 35(2): 478-483.

Soubdhan T, Emilion R, 2010. Stochastic differential equation for modeling global solar radiation sequences. In Proceedings of the IASTED International Conference: Modelling, Identification, and Control (AsiaMIC 2010), 14-17.

Yang D, Jirutitijaroen P, Walsh W, 2012. Hourly solar irradiance time series forecasting using cloud cover index. Solar Energy 86(12): 3531-3543.

Zhou Z, Botterud A, Wang J, Bessa R, Keko H, Sumaili J, Miranda V, 2013. Application of probabilistic wind power forecasting in electricity markets. Wind Energy 16(3): 321-338. 


\section{FIGURES}

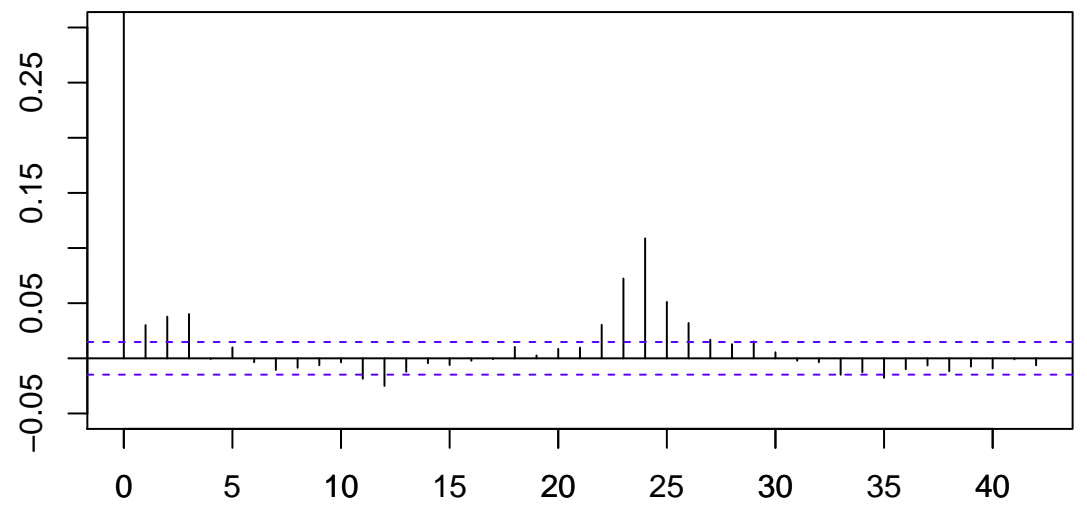

Figure 1. Autocorrelation function for the studentized residuals of Model 2. 

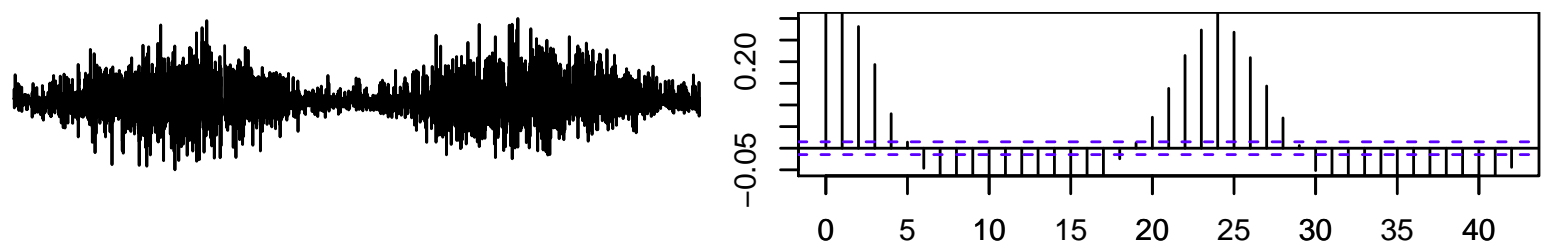

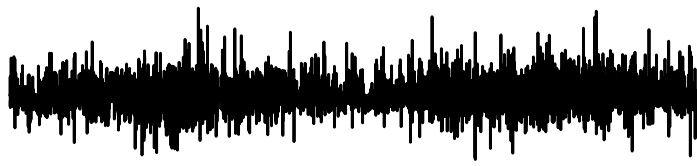
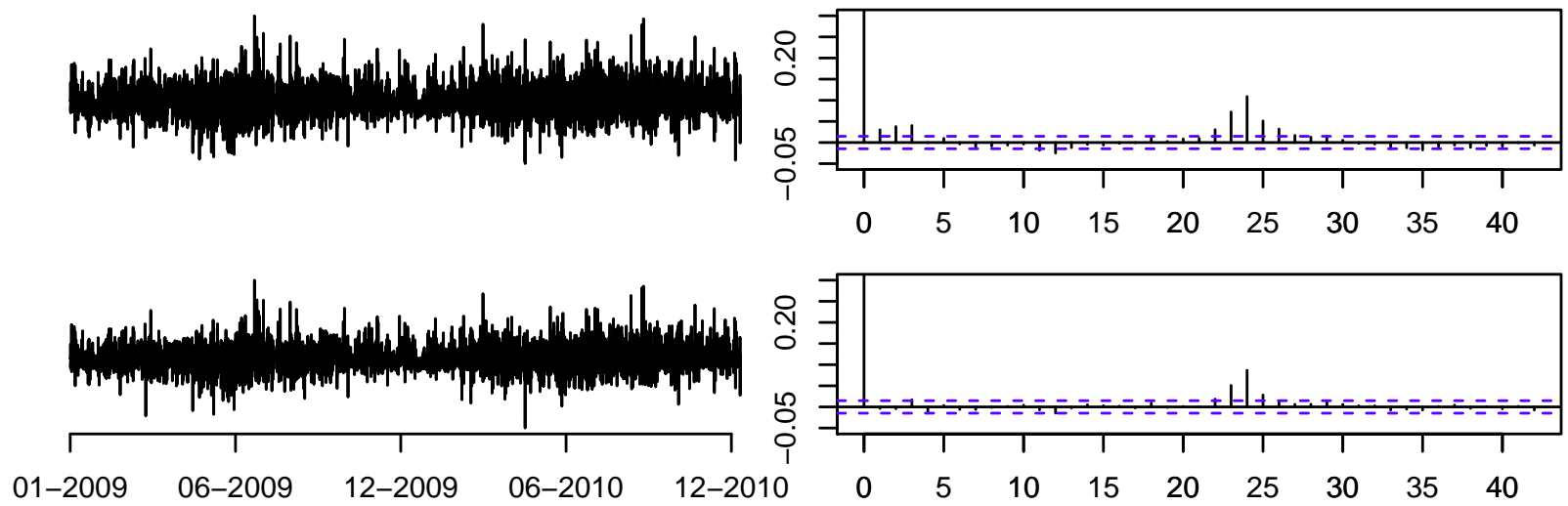

Figure 2. Studentized residuals and autocorrelation function for the residuals. Here the plots in the top line are from Model 1 continueing to the plots in the bottom line from Model 3. 


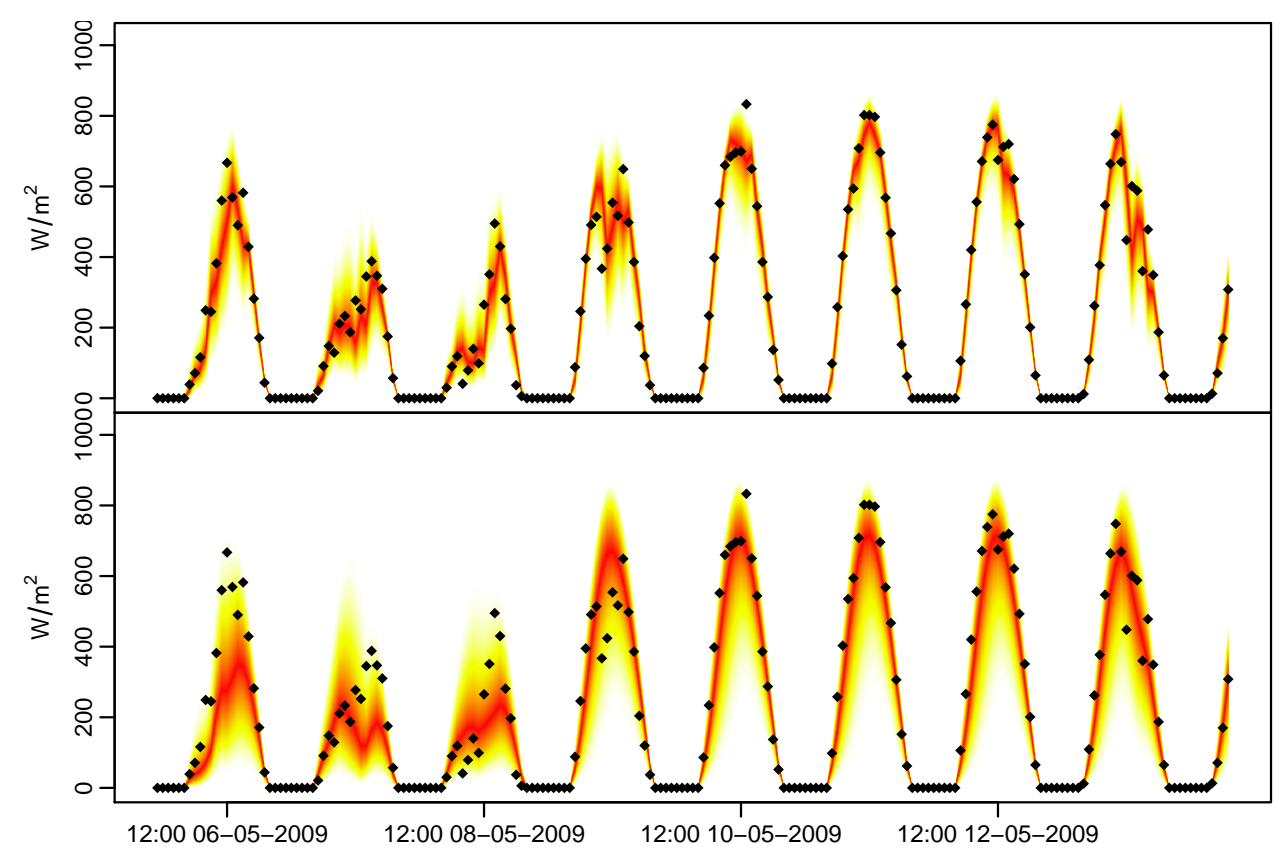

Figure 3. Observations of solar irradiance plotted together with the 1-h ahead predictive densities (top) and 24-h ahead (bottom). Warmer colors indicate a higher probability of seeing this realization. 


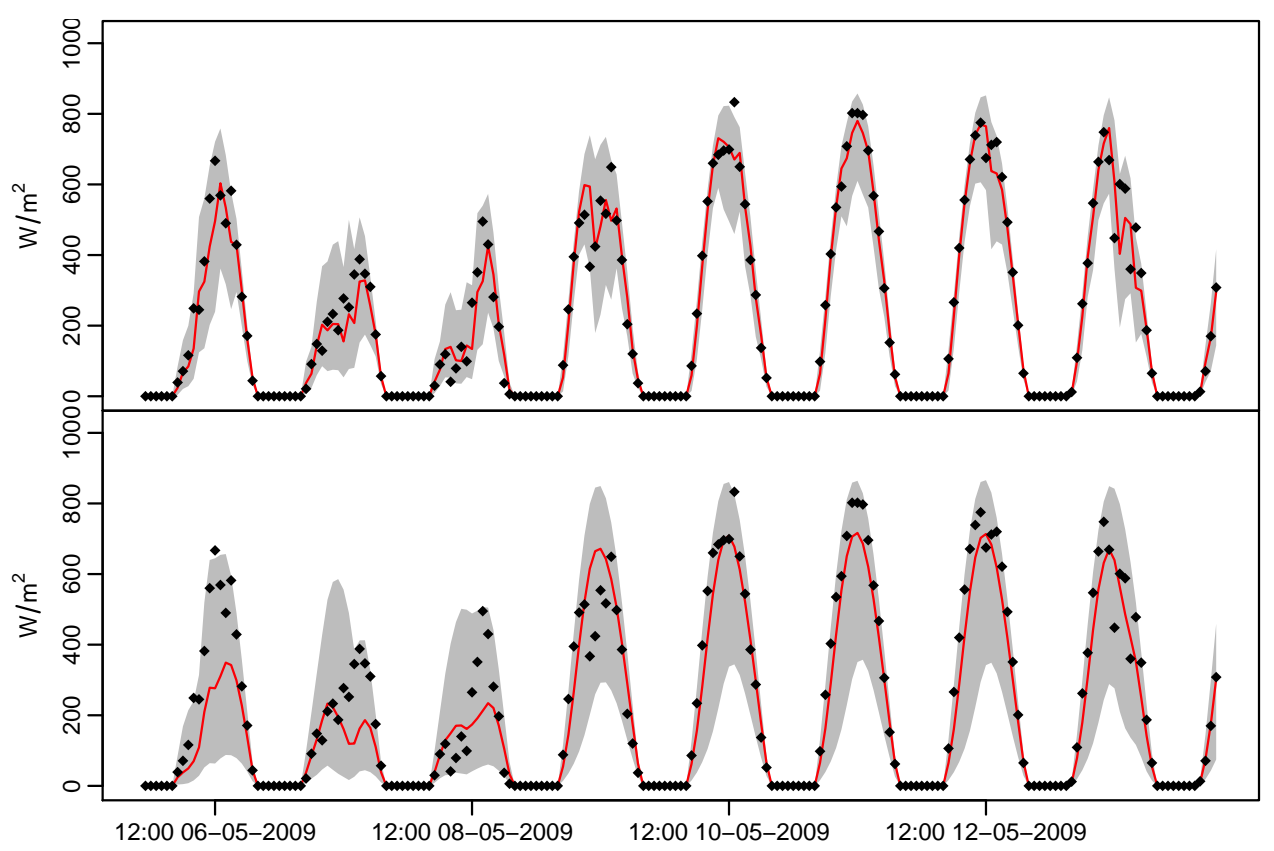

Figure 4. Observations of solar irradiance in black plotted along with the 1-h ahead (top) and 24-h ahead (bottom) $95 \%$ prediction intervals in gray and the prediction in red. 

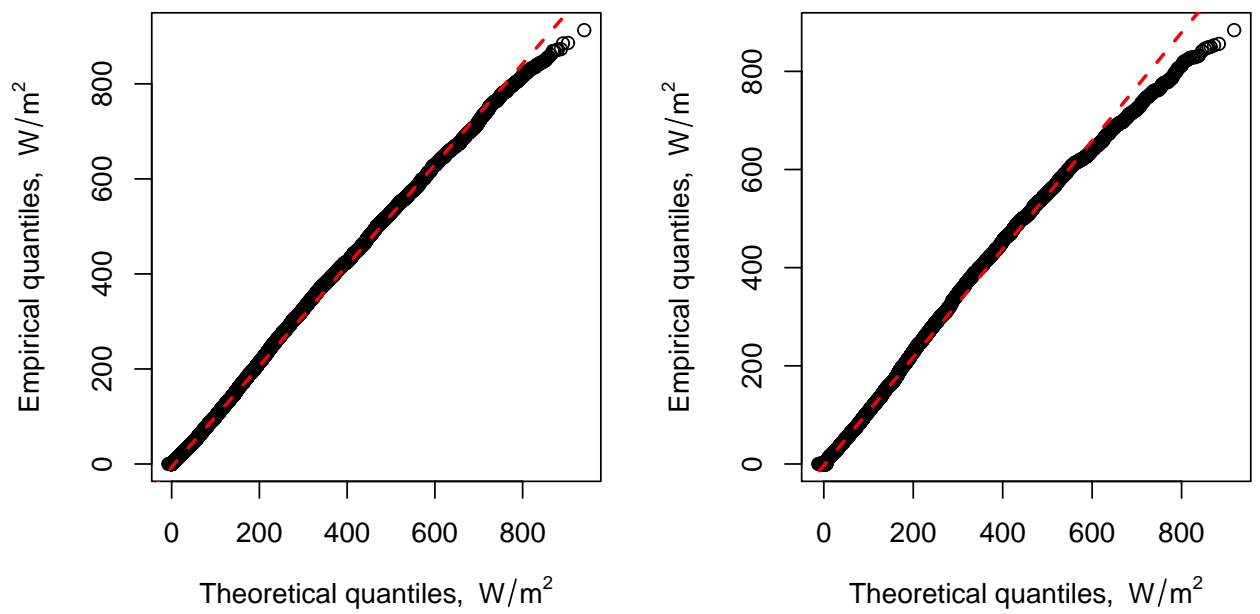

Figure 5. $Q-Q$ plots for the training set (left) and test set (right) based on the empirical data and simulated data from the model over the respective period. 


\section{TABLES}

\begin{tabular}{rrrrrrrrrrrr}
\hline & $\hat{\theta}_{x}$ & $\hat{\mu}_{x}$ & $\hat{\sigma}_{x}$ & $\hat{\sigma}_{\epsilon}$ & $\hat{\beta}_{x}$ & $\hat{\gamma}$ & $\hat{\theta}_{A}$ & $\hat{\mu}_{A}$ & $\hat{\sigma}_{A}$ & $\hat{\omega}_{1}$ & $\hat{\omega}_{2}$ \\
\hline Model 1 & 0.699 & 0.845 & 113 & 0.00101 & - & - & - & - & - & - & - \\
Model 2 & 0.345 & 0.804 & 0.701 & 2.92 & 0.00409 & 0.902 & - & - & - & - & - \\
Model 3 & - & 0.879 & 0.655 & 2.89 & 0.00298 & 0.887 & 1.16 & -1.08 & 1.60 & 0.172 & 0.116 \\
\hline
\end{tabular}

Table 1. Parameter estimates for the different models. 


\begin{tabular}{rrrrrrr}
\hline & & \multicolumn{3}{c}{ Training Set } & & Test Set \\
\cline { 3 - 4 } & d.f. & LL & AIC & BIC & LL \\
\hline Clim.1 & - & -96397 & - & - & -48421 \\
Clim.2 & - & -65060 & - & - & -33801 \\
Clim.3 & - & -48038 & - & - & -24547 \\
AR & 6 & -50218 & - & - & -25172 \\
ARX & 7 & -49024 & - & - & -24567 \\
ARX-GLM & 7 & -46904 & - & - & -23361 \\
Model 1 & 5 & -50286 & 100582 & 100621 & -25230 \\
Model 2 & 7 & -44413 & 88840 & 88894 & -22252 \\
Model 3 & 12 & $\mathbf{- 4 4 1 6 2}$ & $\mathbf{8 8 3 4 8}$ & $\mathbf{8 8 4 4 1}$ & $\mathbf{- 2 2 1 0 2}$ \\
\hline
\end{tabular}

Table 2. In this table the log-likelihood of the different models are shown on the training and test set along with information criteria and degrees of freedom. The climatological predictors are evaluated in terms of empirical likelihood. 


\begin{tabular}{rrrrrrrr}
\hline & & \multicolumn{2}{c}{ Training Set } & & \multicolumn{2}{c}{ Test Set } \\
\cline { 6 - 7 } Quantile function & Expected & $1 \mathrm{~h}$ & $24 \mathrm{~h}$ & & $1 \mathrm{~h}$ & $24 \mathrm{~h}$ \\
\hline$Q(0.1)$ & 0.10 & 0.088 & 0.079 & & 0.076 & 0.061 \\
$Q(0.2)$ & 0.20 & 0.176 & 0.170 & & 0.156 & 0.141 \\
$Q(0.3)$ & 0.30 & 0.273 & 0.261 & & 0.253 & 0.220 \\
$Q(0.4)$ & 0.40 & 0.376 & 0.348 & & 0.349 & 0.301 \\
$Q(0.5)$ & 0.50 & 0.486 & 0.440 & & 0.458 & 0.392 \\
$Q(0.6)$ & 0.60 & 0.603 & 0.540 & & 0.589 & 0.473 \\
$Q(0.7)$ & 0.70 & 0.720 & 0.645 & & 0.712 & 0.580 \\
$Q(0.8)$ & 0.80 & 0.818 & 0.763 & & 0.811 & 0.728 \\
$Q(0.9)$ & 0.90 & 0.901 & 0.885 & & 0.902 & 0.858 \\
\hline
\end{tabular}

Table 3. Frequency of observed exceedances for selected quantiles of the predictive density given by the quantile function $Q(\cdot)$. 